\title{
MAXIMAL HOMOMORPHIC IMAGES OF COMMUTATIVE SEMIGROUPS
}

\author{
by D. B. MCALISTER and L. O'CARROLL \\ (Received 17 January, 1969; revised 11 September, 1969)
}

In this paper we give necessary and sufficient conditions on a commutative semigroup in order that it should have a maximal homomorphic image of one of the following types: (1) groups, (2) semigroups which are unions of groups and (3) pseudoinvertible semigroups, i.e. semigroups having the property that some power of each element lies in a subgroup of the semigroup.

We shall adhere to the terminology and notation of [1]; in particular, we shall use the basic theory of commutative semigroups which is given in [1, Section 4.3].

1. Maximal group homomorphic images. In order to characterise those commutative semigroups which have maximal group homomorphic images, we require the following grouptheoretic lemma.

Lemma 1.1. Let $G$ be a group which has a minimum non-trivial subgroup. Then $G$ is p-primary for some prime $p$.

Proof. Let $K$ be the minimum non-trivial subgroup of $G$. Then $K$ is a group with no non-trivial subgroups and so is cyclic of prime order; say $K$ is generated by $b$, where $b$ has order $p$. If $a$ is a non-identity element of $G$, then, since $K$ is the minimum non-trivial subgroup of $G, b=a^{n}$ for some integer $n$. Thus the subgroup $H(a)$ of $G$, generated by $a$, is a finite commutative group with minimum non-trivial subgroup $K$.

Now, it is immediate from the structure theorem for finite commutative groups [2, Theorem 10.1] that a finite commutative group, which has a minimum non-trivial subgroup, is cyclic of prime power order. Hence $H(a)$ is $p$-primary for each $a \in G$, and so $G$ is p-primary.

THEOREM 1.2. Let $S$ be a commutative semigroup. Then $S$ has a maximal group homomorphic image if and only if the maximal cancellative homomorphic image of $S$ is a group.

Proof. Clearly $S$ has a maximal group homomorphic image if and only if the same is true of its maximal cancellative homomorphic image. Hence it suffices to prove the result for cancellative semigroups.

Let $S$ be a cancellative commutative semigroup, and denote by $G$ the group of quotients of $S$; thus each element of $G$ is of the form $a b^{-1}$ where $a, b \in S$. For each $g \in G$ other than the identity, let $M_{g}$ be a subgroup of $G$ which is maximal with respect to the property of not containing $g$. Let $G_{g}=G / M_{g}$ and denote by $\theta_{g}$ the canonical homomorphism of $G$ onto $G_{g}$. By Lemma 1.1, $G_{g}$ is a periodic group and so $S \theta_{g}$ is a subgroup of $G_{g}$. Since $G$ is generated, as a group, by $S$, it follows that $S \theta_{g}=G_{g}$.

Now suppose that $S$ admits a minimum group congruence; denote this by $\rho$. Then, because each $\theta_{g}$ is a homomorphism of $S$ onto a group, $(a, b) \in \rho$ implies that $a \theta_{g}=b \theta_{g}$ for each 
non-identity element $g \in G$. But, if $a \neq b$, then $a \theta_{g} \neq b \theta_{g}$ for $g=a b^{-1}$. Hence $(a, b) \in \rho$ implies that $a=b$, so that $\rho$ is the identity congruence on $S$ and $S$ is a group.

The converse is obvious.

The proof of Theorem 1.2 shows that a cancellative commutative semigroup admits enough homomorphisms onto groups to separate points. Hence we have the following characterisation of such semigroups.

THEOREM 1.3. Let $S$ be a commutative semigroup. Then $S$ is cancellative if and only if it is a subdirect product of groups.

If $S$ is an $E$-inversive semigroup, then each homomorphic image of $S$ is also $E$-inversive; in particular, the maximal cancellative homomorphic image of $S$ is $E$-inversive. It is clear that a cancellative $E$-inversive semigroup is a group. Hence, by Theorem 1.2 , a commutative $E$-inversive semigroup has a maximal group homomorphic image. Example 1.5 shows that the converse is not true; that is, there exist commutative semigroups which are not $E$-inversive but which have maximal group homomorphic images. However, if a commutative semigroup obeys certain finiteness conditions, for example if it is finitely generated, then it has a maximal group homomorphic image if and only if it is $E$-inversive.

THEOREM 1.4. Let $S$ be a commutative semigroup whose maximal semilattice homomorphic image $\Lambda$ has a minimum member $\alpha$. Then the following are equivalent:

(1) $S_{\alpha}$, the archimedean component of $S$ corresponding to $\alpha$, has an idempotent $e$;

(2) Shas a kernel;

(3) $S$ is E-inversive;

(4) $S$ has a maximal group homomorphic image.

If (1) holds, then Se is the maximal group homomorphic image of $S$.

Proof. (1) $\Rightarrow(2)$. Let $I$ be an ideal of $S$ and let $a \in I$. Then, since $e \in S_{\alpha}$, which is the minimum archimedean class of $S, a e \in S_{\alpha}$. Since $S_{\alpha}$ is archimedean, it follows that $e=e^{n} \in S a e \subseteq I$, for some positive integer $n$. Hence $S e$ is the minimum ideal of $S$.

$(2) \Rightarrow(3)$. If a commutative semigroup has a kernel, then the kernel is a group. Hence, if $S$ has a kernel $K$, for each $a \in S, k \in K$, there exists $x \in K$ such that $a(k x)=(a k) x=e$, where $e$ is the identity of $K$. Thus $S$ is $E$-inversive.

We have already seen that (3) implies (4). Thus, to prove the equivalence of the four statements, it suffices to prove that (4) implies (1).

Suppose that (4) holds, and let $c$ be the minimum cancellative congruence on $S$. Then $S / c$ is, by Theorem 1.2, a group. Hence, for $a \in S_{a}$, there exists $x \in S$ such that $(a, a x a) \in c$. However

$$
c=\{(x, y) \in S \times S: \quad x u=y u \text { for some } u \in S\},
$$

so that $a y=a x a y$ for some $y \in S$. Since $a \in S_{\alpha}, a y$ and $a x$ belong to $S_{\alpha}$ and so it follows, from [5, Proposition 2.4], that $S_{\alpha}$ has an idempotent.

Finally, suppose that (1) holds and denote by $\theta$ the mapping of $S$ onto $S e$ defined by $x \theta=x e$. It is clear that $\theta$ is a homomorphism, and we have already seen that $S e$ is a group. 
Thus $c \subseteq \theta \circ \theta^{-1}$. But, from the definition of $\theta$, we also have $\theta \circ \theta^{-1} \subseteq c$. Hence equality prevails and $S e$ is the maximal group homomorphic image of $S$.

COROLlaRY 1. Let $S$ be a commutative archimedean semigroup. Then the following statements are equivalent:

(1) $S$ has an idempotent $e$;

(2) S has a kernel;

(3) $S$ is pseudoinvertible;

(4) $S$ is E-inversive;

(5) S has a maximal group homomorphic image.

If (1) holds, then Se is the maximal group homomorphic image of $S$.

Proof. By Theorem 1.4, we need only show that (1) is equivalent to (3). In fact, we show that (1) implies (3); it is clear that (3) implies (1).

As in the proof of Theorem 1.4, Se is the kernel of $S$ and so is a group. But, since $S$ is archimedean, if $a \in S$, there exists a positive integer $n$ such that $a^{n} \in S e$. Hence $S$ is pseudoinvertible.

COROLlARY 2. A finitely generated commutative semigroup has a maximal group homomorphic image if and only if it is E-inversive.

Proof. If $S$ is finitely generated, then so is its maximal semilattice homomorphic image $\Lambda$. Hence $\Lambda$ is finite and so has a minimum member.

EXAMPLE 1.5. For each positive integer $n$, let $S_{n}$ be a distinct copy of the additive semigroup of positive integers and let $S=\bigcup\left\{S_{n}: n \geqq 1\right\}$. Then $S$ becomes a commutative semigroup if we define a multiplication as follows: for $x \in S_{m}, y \in S_{n}$,

$$
x y=\left\{\begin{array}{cll}
x+y & \text { if } & m=n \\
x & \text { if } & m>n \\
y & \text { if } & m<n
\end{array}\right.
$$

Clearly $S$ has no idempotents and so, certainly, is not $E$-inversive. However, given $x \in S_{m}, y \in S_{n}$, there exists $z \in S$ such that $x z=y z$; for example, we may take any $z \in S_{m+n}$. It follows that $S / c$ is the trivial group, so that $S$ has a maximal group homomorphic image.

It is known [1, Section 12.1], that there is a free group on any semigroup. That is, if $S$ is a semigroup, then there is a group $G$ and a homomorphism $\gamma: S \rightarrow G$ with the following property: given any homomorphism $\theta$ of $S$ into a group $H$, there exists a unique homomorphism $\psi: G \rightarrow H$ such that the diagram below commutes.

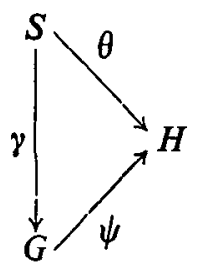


If $S$ is a commutative semigroup then $G$ is the group of quotients of $S / c$ and $\gamma$ is the composite of $c^{4}: S \rightarrow S / c$ with the inclusion mapping $S / c \rightarrow G$. Hence Theorem 1.2 can be stated in the form: if $S$ is a commutative semigroup, then $S$ has a maximal group homomorphic image if and only if $G=S \gamma$.

Even if $S$ is not commutative, the condition $G=S \gamma$ is clearly sufficient in order that $S$ should have a maximal group homomorphic image. On the other hand, the condition $G=S \gamma$ is satisfied in extensive classes of semigroups which are known to admit maximal group homomorphic images; for example, it is satisfied for $E$-inversive semigroups. Hence it is natural to make the conjecture: $S$ has a maximal group homomorphic image if and only if $G=S \gamma$. We end the section by giving an example to show that the conjecture is false.

EXAMPLE 1.6. Let $G$ be a simple group which admits a lattice order; for example, let $G$ be the group $C$ in Theorem 6 of [3]. Let $S$ be the positive cone of $G$. Then each element of $G$ is of the form $a b^{-1}$ where $a, b \in S$, and it can be shown that, if we take $\gamma$ to be the inclusion mapping $S \rightarrow G$, then $G$ is the free group on $S$; if $\theta$ is a homomorphism of $S$ into a group $H$, then $\psi: G \rightarrow H$ is defined by $\left(a b^{-1}\right) \psi=a \theta(b \theta)^{-1}$ for $a, b \in S$.

Suppose that $\theta$ is a homomorphism of $S$ onto a group $H$. Then, since $S$ is not a group, $\theta$ is not one-to-one and therefore neither is $\psi$; thus, since $G$ is simple, $H$ must be the trivial group. Hence $S$ has a maximal group homomorphic image. However $S=S \gamma \neq G$, since $S$ is not a group.

2. Maximal regular homomorphic images. In this section, we shall use Theorem 1.4 to characterise those commutative semigroups which admit maximal regular homomorphic images. To do this, we make use of the decomposition theory for commutative semigroups, which is given in [1, Section 4.3]. For the convenience of the reader, we repeat those details of the theory which we require.

Let $S$ be a commutative semigroup and let $\eta$ be the minimum semilattice congruence on $S$. Then each $\eta$-class is an archimedean semigroup and $S$ is the union of the semilattice $\Lambda \equiv S / \eta$ of semigroups $S_{\alpha}, \alpha \in \Lambda$, where, for each $\alpha \in \Lambda, S_{\alpha}$ is the $\eta$-class corresponding to $\alpha$. This is the unique expression of $S$ as the union of a semilattice of archimedean semigroups.

THEOREM 2.1. Let $S$ be an arbitrary commutative semigroup. Then $S$ has a maximal regular homomorphic image if and only if $S$ is pseudoinvertible.

Let $\sigma$ be the minimum separative congruence on $S$. If $S$ is pseudoinvertible, then $S / \sigma$ is the maximal regular homomorphic image of $S$.

Proof. Suppose that $S$ has a maximal regular homomorphic image, and denote by $\mu$ the minimum congruence $\rho$ on $S$ such that $S / \rho$ is regular. Let $S=\bigcup\left\{S_{\alpha}: \alpha \in \Lambda\right\}$ be the expression of $S$ as the union of a semilattice of archimedean semigroups. Then, for each $\alpha \in \Lambda, \mu_{\alpha}=\mu \cap\left(S_{\alpha} \times S_{\alpha}\right)$ is a congruence on $S_{\alpha}$. We shall show, in a sequence of lemmas, that $\mu_{\alpha}$ is the minimum group congruence on $S_{\alpha}$. By Corollary 1 to Theorem 1.4, this means that each $S_{\alpha}$ is pseudoinvertible. Thus $S$ is pseudoinvertible.

Lemma 2.2. For each $\alpha \in \Lambda, \mu_{\alpha}$ is a group congruence on $S_{\alpha}$. 
Proof. Let $a \in S_{\alpha}$; then, since $S / \mu$ is a regular semigroup, there exists $x \in S$ such that $(a, a x a) \in \mu,(x, x a x) \in \mu$. A semilattice is a regular semigroup; hence $\mu \subseteq \eta$ and so $(a, a x a) \in \eta$, $(x, x a x) \in \eta$. Since $S / \eta$ is a semilattice, it follows that $(a, x) \in \eta$; that is, $x \in S_{\alpha}$. Hence $\mu_{\alpha}$ is a regular congruence on $S_{\alpha}$ and $S_{\alpha} / \mu_{\alpha}$ is a regular semigroup. But $S_{\alpha} / \mu_{\alpha}$ is also archimedean and so has only one idempotent. Thus $S_{\alpha} / \mu_{\alpha}$ is a group.

Let $\rho_{\alpha}$ be a group congruence on $S_{\alpha}$, and pick $u \in S_{\alpha}$ such that $u \rho_{\alpha}^{\natural}$ is the identity of $G=S_{\alpha} / \rho_{\alpha}$. Define $\rho=\rho_{1} \cup \rho_{2}$, where

$$
\rho_{1}=\left\{(x, y) \in D \times D:(x u, y u) \in \rho_{\alpha}\right\}, \quad \rho_{2}=(S \backslash D) \times(S \backslash D)
$$

and

$$
D=\bigcup\left\{S_{\beta}: \beta \geqq \alpha\right\} .
$$

LEMMA 2.3. $\rho$ is a regular congruence on $S$ and

$$
\rho_{\alpha}=\rho \cap\left(S_{\alpha} \times S_{\alpha}\right) \text {. }
$$

Proof. There are two cases according as $\alpha$ is, or is not, the minimum element of $\Lambda$; these correspond to $\rho_{2}=\square$ and $\rho_{2} \neq \square$. In the first case $S / \rho$ is a group and, in the second, $S / \rho$ is a group with zero. We shall give the proof for the second case; the first case is similar.

By [4, Lemma 1.3], $D$ is the complement of a prime ideal of $S$ and, clearly, has minimum archimedean component $S_{\alpha}$. Hence we can define a mapping $\theta$ of $S$ onto $G^{0}$ by

$$
x \theta=\left\{\begin{array}{ccl}
(x u) \rho_{a}^{\natural} & \text { if } & x \in D, \\
0 & \text { if } & x \in S \backslash D .
\end{array}\right.
$$

It is a straightforward matter to verify that $\theta$ is a homomorphism of $S$ onto $G^{0}$ and that $\theta \circ \theta^{-1}=\rho$. Hence $\rho$ is a regular congruence on $S$.

Suppose that $(x, y) \in \rho \cap\left(S_{\alpha} \times S_{\alpha}\right)$; then $(x u) \rho_{\alpha}^{\natural}=(y u) \rho_{\alpha}^{\natural}$ and so, because $u \rho_{\alpha}^{\natural}$ is the identity of $G,(x, y) \in \rho_{\alpha}$. Hence $\rho \cap\left(S_{\alpha} \times S_{\alpha}\right) \subseteq \rho_{\alpha}$. On the other hand, it is clear that $\rho_{\alpha} \subseteq \rho \cap\left(S_{\alpha} \times S_{\alpha}\right)$ so that equality prevails.

LEMMA 2.4. $\mu_{\alpha}$ is the finest group congruence on $S_{\alpha}$.

Proof. Let $\rho_{\alpha}$ be a group congruence on $S_{\alpha}$ and define $\rho$ as above. Then $\rho$ is a regular congruence on $S$ and so $\mu \subseteq \rho$. It follows that

$$
\mu_{\alpha}=\mu \cap\left(S_{\alpha} \times S_{a}\right) \subseteq \rho \cap\left(S_{\alpha} \times S_{\alpha}\right)=\rho_{\alpha} .
$$

Hence $\mu_{\alpha}$ is the minimum group congruence on $S_{\alpha}$.

As remarked before Lemma 2.2, these lemmas show that $S$ is pseudoinvertible if it has a maximal regular homomorphic image. Conversely, if $S$ is pseudoinvertible, then, by [1, p. 135, Question 5], $S / \sigma$ is regular. On the other hand, by [1, Theorem 4.17], a commutative regular semigroup is separative. Hence $\sigma$ is the finest regular congruence on $S$ and $S / \sigma$ is the maximal regular homomorphic image of $S$. 
COROLlaRY. Let $S$ be a commutative semigroup. Then $S$ has a maximal pseudoinvertible homomorphic image if and only if $S$ is pseudoinvertible.

Proof. Suppose that $S$ has a maximal pseudoinvertible homomorphic image and let $\xi$ denote the minimum congruence $\rho$ on $S$ such that $S / \rho$ is pseudoinvertible. By Theorem 2.1, $S / \xi$ has a maximal regular homomorphic image, $T$ say. Since a regular commutative semigroup is pseudoinvertible, $T$ is also the maximal regular homomorphic image of $S$. Hence, by Theorem $2.1, S$ is pseudoinvertible.

The converse is immediate.

\section{REFERENCES}

1. A. H. Clifford and G. B. Preston, The algebraic theory of semigroups, Math. Surveys of the Amer. Math. Soc., Providence, R.I., Vol. 1, 1961; Vol. 2, 1967.

2. L. Fuchs, Abelian groups (Oxford, 1960).

3. C. H. Holland, The lattice ordered group of automorphisms of an ordered set, Michigan Math. J. 10 (1963), 399-408.

4. D. B. McAlister, Characters on commutative semigroups, Quart. J. Math. Oxford Ser. (2) 19 (1968), 141-157.

5. D. B. McAlister and L. O'Carroll, Finitely generated commutative semigroups, Glasgow Math.J. 11 (1970), 134-151.

Department of Mathematics

NORTHERN ILLINOIS UNIVERSITY

DE KALb, IllinOIS, 60115, U.S.A. 